\title{
Communication \\ Comparison of Molecular and In Silico Salmonella Serotyping for Salmonella Surveillance
}

\author{
Linda Chui ${ }^{1,2, *}$, Christina Ferrato ${ }^{3}{ }^{-}$, Vincent $\mathrm{Li}^{2}$ and Sara Christianson ${ }^{4}$ \\ 1 Department of Laboratory Medicine and Pathology, University of Alberta, Edmonton, AB T6G 2R3, Canada \\ 2 Alberta Precision Laboratories-Public Health Laboratory (ProvLab), Edmonton, AB T6G 2J2, Canada; \\ vincent.li@albertaprecisionlabs.ca \\ 3 Alberta Precision Laboratories-Public Health Laboratory (ProvLab), Calgary, AB T2N 4W4, Canada; \\ christina.ferrato@albertaprecisionlabs.ca \\ 4 National Microbiology Laboratory, Public Health Agency of Canada, Winnipeg, MB R3E 3M4, Canada; \\ sara.christianson@canada.ca \\ * Correspondence: linda.chui@albertaprecisionlabs.ca; Tel.: +1-(780)-407-8951
}

Citation: Chui, L.; Ferrato, C.; Li, V.; Christianson, S. Comparison of Molecular and In Silico Salmonella Serotyping for Salmonella Surveillance. Microorganisms 2021, 9, 955. https://doi.org/10.3390/ microorganisms 9050955

Academic Editor: María-Jesús Grilló

Received: 19 March 2021

Accepted: 27 April 2021

Published: 29 April 2021

Publisher's Note: MDPI stays neutral with regard to jurisdictional claims in published maps and institutional affiliations.

Copyright: (c) 2021 by the authors. Licensee MDPI, Basel, Switzerland. This article is an open access article distributed under the terms and conditions of the Creative Commons Attribution (CC BY) license (https:// creativecommons.org/licenses/by/ $4.0 /)$.

\begin{abstract}
Salmonella surveillance and outbreak management is a key function of public health Laboratories are shifting from antigenic serotype determination to molecular methods including microarray or whole genome sequencing technologies. The objective of this study was to compare the Check\&Trace Salmonella ${ }^{\mathrm{TM}}$ DNA microarray (CTS), a commercially available assay with the Salmonella in silico typing resource (SISTR), which uses whole genome sequencing technology for serotyping clinical Salmonella strains in Alberta, Canada, collected over an 18-month period. A high proportion of isolates $(96.3 \%)$ were successfully typed by both systems. SISTR is a powerful tool for laboratories which already have a WGS infrastructure in place, whereas smaller laboratories can benefit from a commercial microarray system and reduce the processing cost per isolate compared to traditional serotyping.
\end{abstract}

Keywords: Salmonella surveillance; serotyping; microarray; whole genome sequencing; public health

\section{Introduction}

Salmonella is a foodborne pathogen and a major source of gastrointestinal infection, with an estimated 93.8 million cases per year [1]. Salmonellosis remains a significant cause of morbidity and mortality, particularly in susceptible populations such as immunocompromised individuals, the elderly, and infants and young children. Public health surveillance reduces the public health burden of salmonellosis by rapidly detecting outbreaks, identifying sources, limiting transmission, and preventing future occurrences. In Canada, PulseNet is a vital surveillance system for pathogens associated with foodborne disease. The Alberta Precision Laboratories-Public Health Laboratory (ProvLab) in Alberta is a PulseNet Canada member that plays an important role in supporting PulseNet surveillance initiatives and outbreak management by serotyping human clinical Salmonella isolates submitted by laboratories across the province. Serotyping is crucial for the rapid and accurate detection of Salmonella outbreaks and is also integral to the resolution process. Historically, Salmonella serotyping was performed using the White-Kauffman-Le Minor (WKL) scheme, which is based on immunological reactions to somatic $(\mathrm{O})$ and flagellar $(\mathrm{H})$ antigens [2]. Although useful, recent technological advances in laboratory diagnostics have produced viable alternatives to conventional serotyping - a test that can be time-consuming, costly, and can produce results that are susceptible to subjective interpretation.

The Check\&Trace Salmonella ${ }^{\mathrm{TM}}$ (CTS) assay (Check-Points, Wageningen, Netherlands) is a commercial DNA microarray system for Salmonella serotyping and is a reliable alternative to conventional methods [3]. The CTS system is based on the targeted amplification and detection of genetic markers followed by hybridization and automated comparison to an 
established database for serotype identification [4]. Serotyping using CTS is rapid and easy to implement in frontline microbiology as well as public health laboratories, and is comparably accurate relative to traditional methods. From 1 March 2015 to 3 February 2020, CTS was the primary method for Salmonella serotyping at the ProvLab.

An increasing number of public health laboratories worldwide have transitioned to next generation sequencing (NGS) for pathogen surveillance [5]. NGS data can be used to assess genetic similarity and evaluate genetic characteristics, such as virulence and antimicrobial resistance [6]. Further, NGS is capable of providing a previously unprecedented level of discrimination between genetically related isolates, and allows for extensive phylogenetic analysis $[7,8]$. These features are particularly useful for Salmonella serotypes that have a high degree of genetic similarity (i.e., Salmonella Enteritidis) and are difficult to differentiate using traditional molecular typing methods, such as pulsed-field gel electrophoresis [9]. Several tools for in silico Salmonella serotype prediction have been developed to utilize the available NGS data and eliminate the necessity of separate serotyping assays [10-12]. One such tool is the Salmonella in silico typing resource (SISTR), which predicts Salmonella serotypes using draft genome sequencing data [13]. SISTR detects the genetic determinants of $\mathrm{O}$ and $\mathrm{H}$ antigens, compares them to an established WKL serotype database, and refines predictions using core genome multi-locus sequencing typing (cgMLST) and phylogenetic analysis.

The evaluation of the different approaches to Salmonella serotyping is important in order to determine the suitability of each assay for routine use in public health laboratories. The purpose of this study is to compare Salmonella serotyping using CTS and SISTR and determine the concordance between the two approaches.

\section{Materials and Methods}

\subsection{Salmonella Strains and CheckETrace Salmonella ${ }^{\mathrm{TM}}$ Molecular Serotyping}

A total of 1397 clinical Salmonella enterica subsp. enterica and non-enterica isolates from Alberta, Canada, submitted to ProvLab from frontline laboratory sites between January 2017 and July 2018, were included in this study. Each isolate represented a unique case of human Salmonella infection in the province. Salmonella isolates were cultured onto a trypticase soy broth agar (TSBA; ProvLab, Edmonton, AB, Canada) and a single colony was selected to perform the Check\&Trace Salmonella assay as per manufacturer's instruction (CheckPoints Rapid Molecular Detection Software v4.9.0.2/2.1.0.19/26-1-2017) and detailed in the publication by Ferrato et al. (2017) [3]. This assay uses a DNA hybridization array system of different known markers, and the software automatically associates known patterns to report a serotype. For reporting purposes, any isolate for which a serotype could not be fully determined by the software was referred to the Public Health Agency of Canada-National Microbiology Laboratory (PHAC-NML, Winnipeg, Manitoba, Canada) to confirm the serotype using conventional methodology, whereby antigenic formulae and serotype determination were based on the White-Kauffmann-Le Minor scheme.

\subsection{DNA Extraction and Genome Sequencing}

A single Salmonella colony was selected and grown overnight in LB-Lennox $0.5 \%$ $\mathrm{NaCl}$ broth for DNA extraction using the Qiagen DNeasy Kit (Qiagen, Valencia, CA, USA) or Epicentre MasterPure Complete DNA and RNA purification Kit (Lucigen, Middleton, WI, USA) as per the manufacturer's instructions. Extracted DNA was quantified using the Qubit ${ }^{\circledR}$ 3.0 Fluorometer (Thermo Fisher Scientific Inc., Mississauga, ON, Canada). Genome sequencing of isolates was performed at the PHAC-NML Core Genomics facility (Winnipeg, MB, Canada). Sample libraries were prepared using the Nextera XT DNA library preparation kit (Illumina, Inc., San Diego, CA, USA), and sequenced using the MiSeq Reagent Kit V3 (600 cycles) and the Illumina MiSeq platform. Data from isolates with a sequencing depth greater than $40 \times$ were included in this study. 


\subsection{In Silico Salmonella Serotyping Using SISTR}

NGS was performed on all 1397 Salmonella isolates and the resulting raw sequence files were uploaded to the Integrated Rapid Infectious Disease Analysis (IRIDA) bioinformatics platform [14]. IRIDA consolidates several bioinformatics analysis pipelines used to process NGS data. In silico serotyping was performed using SISTR as part of the IRIDA platform. The SISTR algorithm is as previously described [13]. Briefly, SISTR assigns Salmonella serotypes using draft genome assemblies by applying a novel 330 loci cgMLST scheme in combination with the characterization of the genetic determinants of $\mathrm{O}-$ and $\mathrm{H}$ - antigens.

\subsection{Data Analysis and Concordance Assessment}

Serotype data assigned by CTS and SISTR platforms were compared and the concordance between the two methods was evaluated. An isolate was considered typed on CTS if there was a match with a serotype in the database. If only a pattern number was generated without associating with a known serotype, the isolate was considered non-typed. Isolates that had discordant results between CTS and SISTR, or that could not be typed by either platform, were conventionally serotyped as described above. Serotypes predicted in silico by SISTR were excluded from the concordance analysis if isolates were missing one or more genes encoding the targeted antigens or if isolates had less than 297 of the 330 loci included in the cgMLST schema. The statistical software SPSS Statistics (IBM) was used to assess the agreement between CTS and SISTR designation for the twenty most frequent Salmonella serotypes.

\section{Results}

Serotyping was performed using SISTR and CTS on all 1397 clinical Salmonella isolates, with confirmation using conventional serotyping as required. These isolates belonged to 87 unique serotypes (77 enterica subspecies; 10 non-enterica subspecies). A total of 1345 $(96.2 \%)$ isolates were successfully serotyped by both CTS and SISTR (Table 1) with only two conflicting results, where a Salmonella (I) 4,[5],12:i:- was typed as $S$. Typhimurium by CTS and a $S$. Virchow was typed as $S$. Javiana by CTS. The distribution of isolates in the 65 different Salmonella serotypes represented is shown in Table 1. S. Enteritidis ( $n=725$; $51.9 \%)$ was the most commonly observed serotype followed by $S$. Typhimurium $(n=101$; $7.2 \%)$, S. Heidelberg $(n=53 ; 3.8 \%)$, and S. Infantis $(n=47 ; 3.4 \%)$. For the 20 most frequent serotypes seen in Alberta throughout this time frame, which represent $87.8 \%$ of all isolates, the agreement between the CTS and SISTR methods was excellent, with $\mathrm{K}=0.983$ (95\% CI $0.973-0.993, p<0.005)$.

Table 1. Results of Salmonella serotyping of 1397 Salmonella strains from human clinical cases using the Check\&Trace Salmonella ${ }^{\mathrm{TM}}$ (CTS) DNA hybridization microarray and Salmonella in silico typing resource (SISTR) method, or by the conventional agglutination method.

\begin{tabular}{|c|c|c|c|c|}
\hline \multirow[t]{2}{*}{ Salmonella Enterica Strains } & \multicolumn{4}{|c|}{ Number of Strains Detected by: } \\
\hline & CTS and SISTR & SISTR & CTS & $\begin{array}{l}\text { Conventional } \\
\text { Agglutination }\end{array}$ \\
\hline \multicolumn{5}{|l|}{ Subsp. Enterica } \\
\hline Enteritidis & 724 & & 1 & \\
\hline Typhimurium & 101 & & & \\
\hline Heidelberg & 53 & & & \\
\hline Infantis & 47 & & & \\
\hline Typhi & 39 & & & \\
\hline (I) 4,[5],12:i:- & $38^{a}$ & & & \\
\hline Newport & 36 & 4 & & \\
\hline Braenderup & 26 & & & \\
\hline
\end{tabular}


Table 1. Cont.

Salmonella Enterica Strains

\begin{tabular}{|c|c|c|c|c|}
\hline & CTS and SISTR & SISTR & CTS & $\begin{array}{l}\text { Conventional } \\
\text { Agglutination }\end{array}$ \\
\hline Javiana & 25 & & & \\
\hline Agona & 22 & & & \\
\hline Reading & 20 & & & \\
\hline Paratyphi B var. Java & 17 & 4 & & \\
\hline Saintpaul & 15 & & & \\
\hline Stanley & 13 & & & \\
\hline Muenchen & 12 & & & \\
\hline Sandiego & 10 & & & \\
\hline Montevideo & 6 & & & 1 \\
\hline Oranienburg & 4 & & 1 & \\
\hline Poona & 4 & 1 & & \\
\hline Indiana & 3 & 1 & & \\
\hline Virchow & $3^{b}$ & 2 & & \\
\hline Adelaide & 1 & 1 & & \\
\hline Give & 1 & 1 & & \\
\hline Apapa & 0 & 1 & & \\
\hline Birkenhead & 0 & 1 & & \\
\hline Carmel & 0 & 1 & & 1 \\
\hline Daytona & 0 & & & 1 \\
\hline Grumpensis & 0 & 1 & & \\
\hline Haifa & 0 & 3 & & \\
\hline Litchfield & 0 & & & 1 \\
\hline Miami & 0 & & & 1 \\
\hline Muenster & 0 & 3 & & \\
\hline Offa & 0 & 2 & & \\
\hline Stanleyville & 0 & 1 & & \\
\hline (I) $4,[5], 12: b:-$ & 0 & 2 & & 4 \\
\hline (I) $42: \mathrm{g}, \mathrm{Z}_{51}:-$ & 0 & & & 1 \\
\hline Other 42 serotypes ${ }^{c}$ & 125 & & & \\
\hline \multicolumn{5}{|l|}{ subsp. salamae } \\
\hline (II) $16: \mathrm{m}, \mathrm{t}:-$ & 0 & & & 1 \\
\hline (II) $48: \mathrm{d}: \mathrm{z}_{6}$ & 0 & 1 & & \\
\hline \multicolumn{5}{|l|}{ subsp. arizonae } \\
\hline (IIIa) $48: z_{4}, z_{24}:-$ & 0 & 2 & & \\
\hline \multicolumn{5}{|l|}{ subsp. diarizonae } \\
\hline (IIIa) $41: z_{4}, z_{23}:-$ & 0 & 1 & & \\
\hline (IIIlb) 60:r:e,n,x,z15 & 0 & 2 & & \\
\hline
\end{tabular}

Number of Strains Detected by: 
Table 1. Cont.

\begin{tabular}{|c|c|c|c|c|}
\hline \multirow[t]{2}{*}{ Salmonella Enterica Strains } & \multicolumn{4}{|c|}{ Number of Strains Detected by: } \\
\hline & CTS and SISTR & SISTR & CTS & $\begin{array}{l}\text { Conventional } \\
\text { Agglutination }\end{array}$ \\
\hline (IIIlb) 60:r:z & 0 & & & 1 \\
\hline (IIIb) 65:k:z & 0 & 1 & & \\
\hline (IIIb) O Rough:c:z & 0 & & & 1 \\
\hline \multicolumn{5}{|l|}{ subsp. houtenae } \\
\hline (IV) $43: z_{4}, z_{23}:-$ & 0 & 1 & & \\
\hline TOTAL number of strains & 1345 & 37 & 2 & 13 \\
\hline
\end{tabular}

${ }^{a}$ CTS result was $S$. Typhimurium for one isolate. ${ }^{b}$ CTS result was $S$. Javiana for one isolate. ${ }^{c}$ Other $S$. enterica subsp. enterica isolates detected less than 10 times and showing the same serotype from both CTS and SISTR methods.

In this study, 37 isolates could not be serotyped by CTS, 2 isolates could not be serotyped by SISTR, and 13 isolates failed to be serotyped by both platforms, so conventional methods were used to determine these serotype (Table 1). The 15 isolates that could not be serotyped using SISTR were distributed across 12 different serotypes. A total of 21 serotypes were identified from the 37 isolates typed by SISTR but not CTS, and all of these isolates were concordant with conventional typing results. Of the 13 isolates that could not be serotyped by both SISTR and CTS, S. enterica subspecies enterica (I) 4,[5],12:b:(possible Paratyphi B var Java, monophasic d-tartrate positive) was the most common serotype $(n=4)$ identified using the conventional method. Furthermore, these four isolates shared the same CTS microarray pattern.

\section{Discussion}

Rapid and accurate serotyping is crucial for Salmonella public health surveillance and outbreak management. Serotyping using CTS and the in silico serotype prediction tool SISTR was evaluated, and the two approaches were compared. Of the isolates typed by both assays, a high degree of concordance in the serotype assignments (99.9\%; Table 1) was observed across a wide range of different serotypes, as only two isolates were discordant between the two methods. A total of $964(69.0 \%)$ isolates included in this study were identified as one of the top five most common Salmonella serotypes seen in Canada [15]: Enteritidis, Typhimurium, (I) 4,[5],12:i:-, Heidelberg, and Infantis. Of these isolates, 963 $(99.9 \%)$ were concordantly identified by CTS and SISTR. The one discordant isolate was $S$. Enteritidis, and this could not be serotyped by SISTR because the $f l i C$ gene encoding the H1 antigen could not be detected. Individually, both CTS (1346/1397; 96.3\%) and SISTR $(1382 / 1397 ; 98.9 \%)$ were able to accurately serotype the vast majority of isolates, and identified many uncommon serotypes (Table 1). The success rate observed for SISTR in this study is higher than previously reported values (94.8\%) [8].

In total, $52(3.8 \%)$ of the 1397 isolates included in this study could not be serotyped using CTS $(n=37)$, SISTR $(n=2)$, or both $(n=13)$. Of these, 11 isolates were identified as non-enterica subspecies. CTS was unable to serotype any of these 11 isolates; however, SISTR was able to identify the serotypes for 8 (Table 1). Several of the remaining isolates that CTS and/or SISTR had difficulty serotyping belong to rare subsp. enterica serotypes.

The ability of CTS to identify rare serotypes will be improved as the CTS database becomes more populated. Many non-typed isolates of the same serotype achieved the same numerical microarray pattern number, and so progressive updates to the CTS database (or local experience) can allow users to serotype isolates that had no previous association to a serotype in the software. In this study, four $S$. Newport and three $S$. Haifa isolates fell into this category (Table 1). For the three $S$. Haifa isolates, CTS detected differences in marker presence, but the serotypes assigned by the current version of the CTS software do not reflect the presence of these markers. Future updates of the CTS database and software 
will eliminate this discrepancy. Laboratories using CTS can also internally validate specific microarray patterns in order to report serotypes beyond the software update they are using.

A similar issue is observed when using SISTR as the cgMLST schema, whereby analysis relies on phylogenetic comparison to a serotype reference database [13]. The extent that rare serotypes and genetic diversity are represented in the SISTR database affects the serotype predictive ability of the algorithm. In this study, 15 isolates could not be serotyped by SISTR. Of these, 14 failed because they did not meet the minimum number of loci required for cgMLST-based serotype prediction. When the minimum cgMLST threshold score was less stringent, 11 of these 14 isolates had serotypes in agreement with CTS and/or conventional serotyping. Genome assembly quality also has a significant impact on the cgMLST threshold scores, and consequently the ability of SISTR to accurately predict the Salmonella serotypes [13]. A limitation of SISTR that will likely persist is its inability to confidently serotype isolates with partial antigenic expression. Monophasic serotype variants of $S$. Typhimurium are problematic for SISTR [16], and one discordant falls into this category. These isolates will require the additional characterization of individual antigens using conventional methods. However, it is important to note that isolates that fail to express an antigen remain problematic, and this is an underlying issue associated with relying on serotyping alone to identify genetic relationships between isolates. Serotyping is also prone to identifying spurious relationships. For these reasons, the cgMLST-based clustering algorithm used by SISTR should be the gold standard for determining relatedness, and the serotype should only be used as a screening tool. Serotyping results are an early indicator for potential clusters, alerting epidemiologists of situations wherein public health action may be necessary. The results of cgMLST-based clustering provide confirmation of suspect cluster cases and prompt further outbreak management.

There were two $S$. Virchow by SISTR that were not typed by CTS and were confirmed as $S$. enterica subspecies enterica (I) 6,7:r:-, a monophasic variant of $S$. Virchow, by conventional typing. For these two isolates, there were differences between the serotypes predicted by SISTR using cgMLST (S. Virchow) and antigen data (S. enterica subspecies enterica (I) 6,7:r:-). Since the SISTR algorithm favors the cgMLST schema when there is a discrepancy, $S$. Virchow was assigned to the two isolates. However, the serotype designation from the algorithm used by SISTR to infer antigen genes based on serogroup determination matched the antigenic formula achieved by conventional methods. This highlights the possibility of differences in serotype designation arising from shifts in the serotyping paradigm between different methods. For these reasons, traditional serotyping will still be necessary to identify the serotypes for isolates that could not be typed with confidence in silico.

Both CTS and SISTR serotyping are effective alternatives to conventional serotyping methods, which require technical expertise and quality control for the many antisera. The cost of performing serotyping using the CTS system is approximately CAD 60 per isolate, and is suitable for smaller laboratories that may not have access to capital for NGS equipment and bioinformatics pipelines for analysis, or do not have enough specimen volume to justify the upfront cost of NGS. Further, the hands-on processing and analysis for SISTR is labor-intensive and requires trained expertise, with the entire process taking a minimum of $4-5$ days to complete. CTS features automated analysis with a practical turn-around time of $24 \mathrm{~h}$ from the isolation of the organism to reporting the serotype result, and it requires less technical expertise to operate.

Overall, both methods were largely successful at serotyping the Salmonella isolates in this study, and can drastically decrease the time and cost per isolate by providing an alternative method to conventional serotyping. SISTR is particularly useful if NGS data are readily available; however, more time requirement and expertise are needed to perform the analysis, while CTS produces comparable results without the high initial costs in equipment and staffing associated with NGS. In laboratories that are set up for NGS, the sequencing data used by the SISTR pipeline can also be analyzed to explore genomes for cluster detection, antibiotic resistance and other virulence gene markers, including the 
discovery of new genes. Although SISTR is not an ideal technique for detecting outbreaks or clusters in a timely manner as compared to the CTS system, it does provide an excellent platform to collect meta data for understanding the nature of the isolates. Both methods drastically reduce the time and cost per sample for ongoing serotyping compared to conventional methods.

Author Contributions: Conceptualization, L.C.; methodology, S.C.; software, V.L.; validation, V.L. and C.F.; formal analysis, L.C., C.F. and V.L; resources, L.C.; data curation, V.L.; writing-original draft preparation, V.L.; writing—review and editing, C.F., L.C., S.C.; supervision, L.C. All authors have read and agreed to the published version of the manuscript.

Funding: This research received no external funding.

Acknowledgments: The authors would like to thank the staff at Alberta Precision LaboratoriesPublic Health Laboratory (ProvLab) and the National Microbiology Laboratory's Division of Enteric Diseases (Reference and Diagnostic Services) as well as PulseNet Canada. Special thanks to Bonita Lee (Alberta Health Services) for input on some aspects of the analysis.

Conflicts of Interest: The authors declare no conflict of interest.

\section{References}

1. Majowicz, S.E.; Musto, J.; Scallan, E.; Angulo, F.J.; Kirk, M.; O’Brien, S.J.; Jones, T.F.; Fazil, A.; Hoekstra, R.M. International collaboration of enteric disease "Burden of Illness" studies. The global burden of nontyphoidal Salmonella gastroenteritis. Clin. Infect. Dis. 2010, 50, 882-889. [CrossRef]

2. Guibourdenche, M.; Roggentin, P.; Mikoleit, M.; Fields, P.I.; Bockemuhl, J.; Grimont, P.A.D.; Weill, F.X. Supplement $2003-2007$ (No. 47) to the White-Kauffmann-Le-Minor scheme. Res. Microbiol. 2010, 161, 26-29. [CrossRef]

3. Ferrato, C.; Chui, L.; King, R.; Louie, M. Utilization of a molecular serotyping method for Salmonella enterica in a routine laboratory in Alberta Canada. J. Microbiol. Methods 2017, 135, 14-19. [CrossRef] [PubMed]

4. Wattiau, P.; Weijers, T.; Andreoli, P.; Schliker, C.; Veken, H.V.; Maas, H.M.E.; Verbruggen, A.J.; Heck, M.E.O.C.; Wannet, W.J.; Imberechts, H.; et al. Evaluation of the Premi Test Salmonella, a commercial low-density DNA microarray system intended for routine identification and typing of Salmonella enterica. Int. J. Food Microbiol. 2008, 123, 293-298. [CrossRef]

5. Deng, X.; den Bakker, H.C.; Hendriksen, R.S. Genomic Epidemiology: Whole-genome-sequencing-powered surveillance and outbreak investigation of foodborne bacterial pathogens. Annu. Rev. Food Sci. Technol. 2016, 7, 353-374. [CrossRef] [PubMed]

6. Pornsukarom, S.; van Vliet, A.H.M.; Thakur, S. Whole genome sequencing analysis of multiple Salmonella serovars provides insights into phylogenetic relatedness, antimicrobial resistance, and virulence markers across humans, food animals and agriculture environmental sources. BMC Genom. 2018, 19, 801. [CrossRef]

7. Gilchrist, C.A.; Turner, S.D.; Riley, M.F.; Petri, W.A., Jr.; Hewlett, E.L. Whole-genome sequencing in outbreak analysis. Clin. Microbiol. Rev. 2015, 28, 541-563. [CrossRef] [PubMed]

8. Yachison, C.A.; Yoshida, C.; Robertson, J.; Nash, J.H.E.; Kruczkiewicz, P.; Taboada, E.N.; Walker, M.; Reimer, A.; Christianson, S.; Nichani, A.; et al. The validation and implications of using whole genome sequencing as a replacement for traditional serotyping for a national Salmonella reference laboratory. Front. Microbiol. 2017, 9, 1044. [CrossRef] [PubMed]

9. den Bakker, H.C.; Allard, M.W.; Bopp, D.; Brown, E.W.; Fontana, J.; Iqbal, Z.; Kinney, A.; Limberger, R.; Musser, K.A.; Shudt, M.; et al. Rapid whole-genome sequencing for surveillance of Salmonella enterica serovar enteritidis. Emerg. Infect. Dis. 2014, 20, 1306-1314. [CrossRef] [PubMed]

10. Zhang, S.; Yin, Y.; Jones, M.B.; Zhang, Z.; Deatherage Kaiser, B.L.; Dinsmore, B.A.; Fitzgerald, C.; Fields, P.I.; Deng, X. Salmonella serotype determination utilizing high-throughput genome sequencing data. J. Clin. Microbiol. 2015, 53, 1685-1692. [CrossRef]

11. Tewolde, R.; Dallman, T.; Schaefer, U.; Sheppard, C.L.; Ashton, P.; Pichon, B.; Ellington, M.; Swift, C.; Green, J.; Underwood, A. MOST: A modified MLST typing tool based on short read sequencing. Peer] 2016, e2308. [CrossRef] [PubMed]

12. Zhang, S.; den Bakker, H.C.; Li, S.; Chen, J.; Dinsmore, B.A.; Lane, C.; Lauer, A.C.; Fields, P.I.; Deng, X. SeqSero2: Rapid and improved Salmonella serotype determination using whole-genome sequencing data. Appl. Environ. Microbiol. 2019, 85, e01746-19. [CrossRef] [PubMed]

13. Yoshida, C.E.; Kruczkiewicz, P.; Laing, C.R.; Lingohr, E.J.; Gannon, V.P.J.; Nash, J.H.E.; Taboada, E.N. The Salmonella In Silico Typing Resource (SISTR): An open web-accessible tool for rapidly typing and subtyping draft Salmonella genome assemblies. PLoS ONE 2016, 11, e0147101. [CrossRef] [PubMed]

14. Integrated Rapid Infectious Disease Analysis (IRIDA). Available online: www.irida.ca (accessed on 15 August 2018 ).

15. Government of Canada. National Enteric Surveillance Program. (NESP) Annual Summary 2019; Public Health Agency of Canada: Guelph, ON, Canada, 2020.

16. Robertson, J.; Yoshida, C.; Kruczkiewicz, P.; Nadon, C.; Nichani, A.; Taboada, E.N.; Nash, J.H.E. Comprehensive assessment of the quality of Salmonella whole genome sequence data available in public sequence databases using the Salmonella in silico Typing Resource (SISTR). Microb. Genom. 2018, 4, e000151. [CrossRef] [PubMed] 